\title{
Efficacy and Safety of Berberine for Prediabetes: A Systematic Evaluation and Meta-Analysis
}

\author{
Li Wang1, Guohong Wei², Longyun Peng 3 , Hui Ge ${ }^{1 *}$ \\ ${ }^{1}$ Department of Healthcare, The First Affiliated Hospital, Sun Yat-sen University, Guangzhou, China \\ ${ }^{2}$ Department of Endocrinology, The First Affiliated Hospital, Sun Yat-sen University, Guangzhou, China \\ ${ }^{3}$ Department of Cardiology, The First Affiliated Hospital, Sun Yat-sen University, Guangzhou, China \\ Email: *gehui2009gh@126.com
}

How to cite this paper: Wang, L., Wei, G.H., Peng, L.Y. and Ge, H. (2021) Efficacy and Safety of Berberine for Prediabetes: A Systematic Evaluation and Meta-Analysis. International Journal of Clinical Medicine, 12, 131-144.

https://doi.org/10.4236/ijcm.2021.124014

Received: March 22, 2021

Accepted: April 16, 2021

Published: April 19, 2021

Copyright ( 2021 by author(s) and Scientific Research Publishing Inc. This work is licensed under the Creative Commons Attribution International License (CC BY 4.0).

http://creativecommons.org/licenses/by/4.0/

\begin{abstract}
Objective: To assess the efficacy and safety of berberine in the treatment of prediabetes. Methods: We searched the following databases, CNKI, WanFang, VIP, CBM, PubMed, Cochrane Library, Embase, and Medline (OVID) from the databases established to December 2020 in Chinese or English language. Randomized control trials (RCTs) of berberine compared with lifestyle modification, placebo, and/or hypoglycaemics intervention on treating prediabetes were included. Data extraction and paper quality assessment were conducted according to the Cochrane Handbook. RevMan 5.4 was used for the meta-analysis. Results: Seven studies involving 859 participants were included in the study and the control groups were all lifestyle modification or metformin treatment. The clinical heterogeneity of the trials was relatively high, and the methodological quality of most trials was generally low. Meta-analysis suggested that berberine could reduce FPG $(P=0.001), 2 \mathrm{hPG}(P=$ $0.001)$ and HbA1c $(P=0.002)$ levels significantly as compared with lifestyle group. There was no statistical significance between berberine and metformin. No serious adverse effects from berberine were reported. Conclusions: Berberine has good efficacy and safety in the treatment of prediabetes. Due to the quality limitations of the included trials, the above conclusions need to be further verified by high-quality, large sample size and multi-center clinical trials.
\end{abstract}

\section{Keywords}

Berberine, Prediabetes, Efficacy, Safety, Meta-Analysis

\section{Introduction}

Prediabetes refers to the intermediate hyperglycemia state between normal blood 
glucose and diabetes [1]. It is also known as impaired glucose regulation (IGR), including impaired fasting glucose (IFG) and/or impaired glucose tolerance (IGT). The epidemiological survey showed that the prevalence of prediabetes was $35.2 \%$ [2]. The prevalence among males and females was $37.0 \%$ and $33.4 \%$ respectively [2]. Prediabetes is considered the most significant risk factor for type 2 diabetes (T2DM). It is estimated that the world's prediabetic population will grow to 470 million by 2030 [3], and about $70 \%$ of them will develop diabetes at some point [4]. The damages of hyperglycemia already exist before diabetes. So prediabetes is considered a marker or watershed, which means that the risk of cardiavascular disease, diabetes, microangiopathy, tumor, and dementia will increase in the future [5]. But the blood sugar can be reversed to normal after appropriate treatment. At Present, prediabetic patients mainly depend on lifestyle interventions to prevent or delay diabetes. It is generally considered to be safe and cost-effective. However, only a small number of patients can adhere to their diatary plans and exercise prescriptions and it is reported that about $10 \%-20 \%$ of prediabetic patients are resistant to the effects of exercise with weight loss [6]. A six-year follow-up study showed that about $50 \%$ of the prediabetic patients who had received lifestyle interventions still developed diabetes [7]. Therefore, the guidelines recommend if no satisfactory results are achieved after six months of active interventions, metformin or acarbose should be considered [5]. For young patients having solid financial support and strong health needs, early pharmaceutical interventions are highly recommended [8]. But the high cost and side effects of western medician limit the clinical application. So it becomes increasingly important to develop cost-effective safe hypoglycemic drugs.

Prediabetes belongs to the "spleen" of traditional Chinese medicine (TCM), which is the philosophy of corrective and preventative action against disease [9] [10]. Berberine (BBR, molecular formula: C20H19NO5, molecular weight: 353.36) is a natural alkaloid extracted from the rhizome of Chinese goldthread (Coptis chinensis) and Phellodendron bark (Cortex phellodendri) [11] and is well known as the effective drug that can relieve the symptoms of infectious diarrhea. Modern pharmacological studies have confirmed that berberine has significant hypoglycemic and lipid-regulating effects, improving insulin resistance and anti-inflammatory effects [12] [13] [14]. Some clinical trials have also confirmed berberine has the same hypoglycemic effect on prediabetes. But no one has done a systematic evaluation for it. This research used the Cochrane systematic evaluation method and evaluated the efficacy and safety of berberine in treating prediabetes in RCTs. This can provide a critical reference for clinical decision-making.

\section{Materials and Methods}

\subsection{Search Strategy}

We searched the China National Knowledge Infrastructure (CNKI), the Wan- 
Fang Database, the Chinese Scientific Journal Database (VIP), the Chinese BioMedical Literature Database (CBM), PubMed, Cochrane Library, Embase, and Medline (OVID) from the databases established to December 2020 in any language. Ongoing trials reported by ClinicalTrials.gov were also searched. The following search terms were used: ["Berberine" or "Huangliansu" or "Xiaopojian"] and ["prediabetes" or "pre-diabetes" or "impaired glucose tolerance" or "impaired fasting glucose" or "impaired glucose regulation"]. In addition, the reference lists from articles were manually searched for further studies.

\subsection{Inclusion Criteria}

Studies were included if they fulfilled the following criteria: design of parallel RCT of berberine intervention compared with lifestyle modification, placebo, and/or hypoglycaemics on treating prediabetes, whether allocation concealment and blinding were used or not; Literature is either Chinese or English literature. Some studies contained multiple groups and each comparison group containing berberine was considered as a separate trail in the analysis. Studies were only included if the intervention was given for at least 2 months. Prediabetes was diagnosed by internationally recognized criteria. No sex or age limitation. The diagnosis criteria include WHO 1999 [15], CDS 2013 [16] and ADA 2010 [17].

The primary outcomes consisted of fasting plasma glucose levels (FPG), 2-hour postprandial plasma glucose (2hPG), glycosylated haemoglobin levels A1c (HbA1c) and homeostasis model assessment of insulin resistance (HOMA-IR), homeostasis model assessment of $\beta$ cell function (HOMA- $\beta$ ). The secondary outcomes consisted of body mass index (BMI) and adverse effects.

\subsection{Exclusion Criteria}

The exclusion criteria were non-randomized controlled trials and quasirandomized control trials; abstracts or comments from conference papers; animal studies or comparative studies on different Chinese medicine therapies.

\subsection{Data Extraction}

Literature selecting: read the article title and abstract, eliminated the studies not meeting the inclusion/exclusion criteria. Two reviewers independently assessed trials for inclusion in the review. They extracted data concerning details of the sample size, interventions, duration of treatment, and outcomes by using a standard Microsoft Excel (Microsoft Corporation, office 2016) file. Any disagreements were resolved by consensus, or if required by a third reviewer.

\subsection{Quality Assessment}

The quality of the included trials was assessed using the Cochrane risk bias tools (Review Manager 5.4 provided by the Cochrane Collaboration) [18]. The criteria include random sequence generation, allocation concealment, blinding of participants and personnel, blinding of outcome assessment, incomplete outcome 
data, selective reporting and other bias. We made judgement on each of these criteria relating to the risk of bias: low, high, or unclear (indicating unclear or unknown risk of bias).

\subsection{Statistical Methods}

We used the RevMan 5.4 meta-analysis software to summarize the effects of berberine. Categorical variables used odds ratio (OR) and continuous variables used the mean differences (MD) as analysis statistics. 95\% confidence interval (95\% CI) was used as the effective size for the combined analysis. The clinical and methodological heterogeneity of the included studies was evaluated with $X^{2}$ test and $\vec{F}$ test. The different berberine interventions and control methods were used for sensitivity subgroup analysis. Reporting bias was explored through funnel plot analysis when the number of included trials exceeded ten. A fixed-effect model was used when the studies in the subgroup were sufficiently similar $\left(I^{2}<\right.$ $50 \%, P>0.10)$. Otherwise, a random-effect model was used. When $P<0.05$, it indicated that there was a significant difference between the two groups. Interval estimation and hypothesis test results were shown in the forest plot.

\section{Results}

\subsection{Literature Search Results}

The flowchart of study search results is displayed in Figure 1. The primary searches identified a total of 364 references. 153 articles were screened after 211 duplicates of the same articles were removed. According to the inclusion criteria, 146 records were excluded because they were animal studies, not prediabetes, not RCTs, reviews or comments. Finally, seven studies met the eligibility criteria and were included in the systematic review and meta-analysis.

\subsection{Characteristics of the Included Studies}

The seven studies, including six in Chinese and one in English, were published in 2007-2020. All the studies were performed as single center trials and originated from the mainland of China. Six studies [19] [20] [22] [23] [24] [25] adopted two-armed paralled group design. One study [21] adopted three-armed group design, including berberine, lifesyle modification and metformin. One study [23] set a washout period between two treatment periods of berberine vs. lifestyle modification. According to the inclusion criteria, the two studies [21] [23] were analyzed as four trials. A total of 859 prediabetic patients were enrolled. Among them, 431 were in the experimental group and 428 in the control. The baseline consistency of each trial was comparable. See Table 1.

\subsection{Risk of Bias in Included Studies}

We used RevMan 5.4 to assess the risk of bias in included seven studies. None of them reported the research plan and sample size estimation method. All the studies mentioned random assignment of participants. But only two studies described 


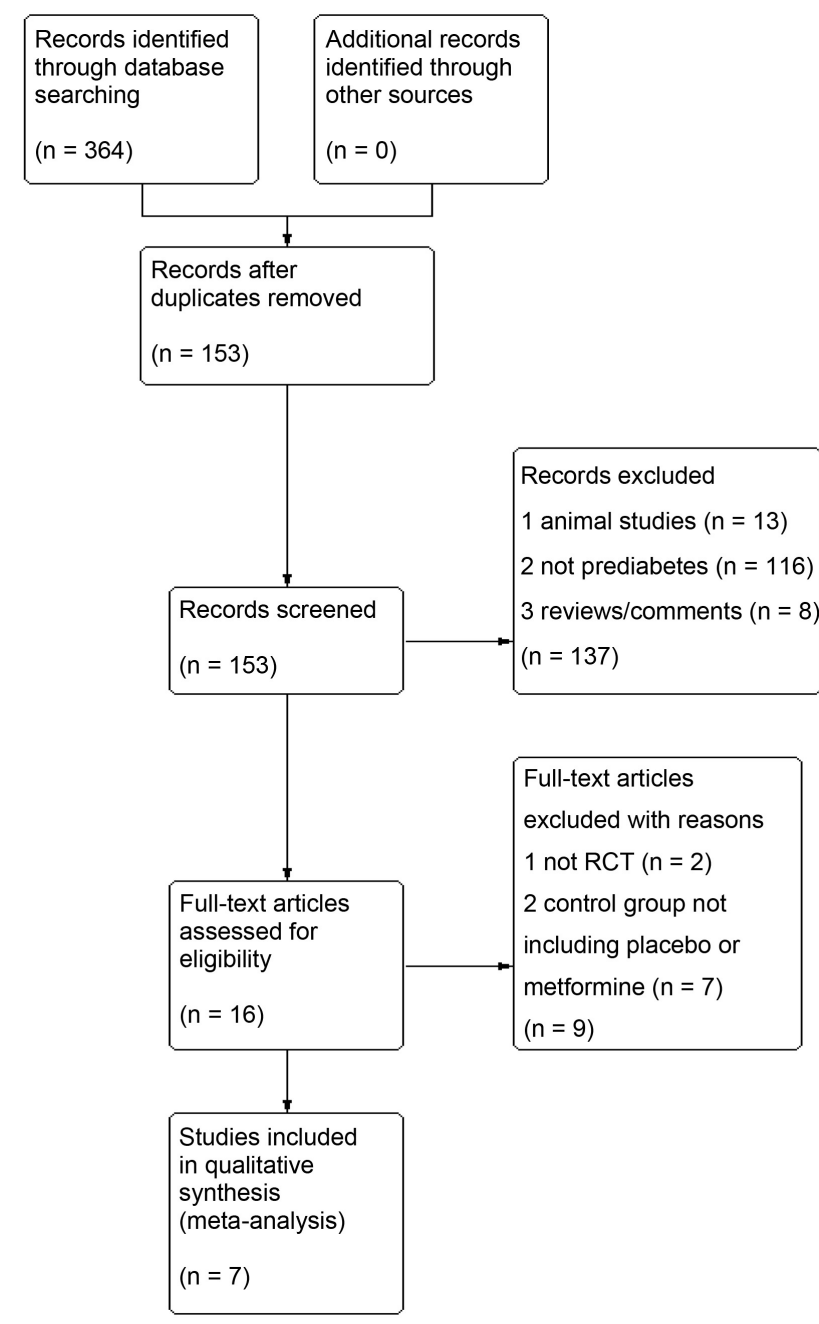

Figure 1. Flow chart of the strategy used for the selection of studies used in the meta-analysis.

Table 1. Characteristics of the included trials.

\begin{tabular}{|c|c|c|c|c|c|c|}
\hline \multirow{2}{*}{ Study } & \multicolumn{2}{|c|}{ Number of patients (Man/Female) } & \multicolumn{2}{|c|}{ Intervation } & \multirow{2}{*}{$\begin{array}{l}\text { Duration } \\
\text { (month) }\end{array}$} & \multirow{2}{*}{ Outcomes } \\
\hline & Experimental & Control & Experimental & Control & & \\
\hline Ju SB 2007 [19] & $46(28 / 18)$ & $44(26 / 18)$ & BBR $0.6 / d+L M$ & LM & 12 & (1)(2)(3)(4)(6) \\
\hline Zhang ZJ 2018 [20] & $50(27 / 23)$ & $50(32 / 18)$ & BBR $0.3 \mathrm{tid}+\mathrm{LM}$ & LM & 4 & (1)(6) 7 ) \\
\hline Chang HY 2020 (1) [21] & $80(45 / 35)$ & $80(42 / 38)$ & BBR $30 \mathrm{mg}$ tid & LM & 6 & (1)(2)(3)(4)(6)(7) \\
\hline Chang HY 2020 (2) [21] & $80(45 / 35)$ & $80(41 / 39)$ & BBR $30 \mathrm{mg}$ tid & Met 0.25 tid $\rightarrow 0.5$ tid & 6 & (1)(2)(3)(4)(6) (7) \\
\hline Zhang Z 2020 [22] & $24(-/-)$ & $24(-/-)$ & BBR $0.2 \mathrm{tid}+\mathrm{LM}$ & LM & 2 & (6) \\
\hline Wang L 2020 (1) [23] & $35(19 / 16)$ & $35(19 / 16)$ & BBR 0.3 tid + LM & LM & 3 & (1)(2)(3)(4)(7) \\
\hline Wang L 2020 (2) [23] & $34(19 / 15)$ & $33(18 / 15)$ & BBR $0.3 \mathrm{tid}+\mathrm{LM}$ & LM & 3 & (1)(2)(3)(4)(7) \\
\hline Zhao JQ 2018 [24] & $32(26 / 6)$ & $32(24 / 8)$ & BBR $0.3 \mathrm{tid}+\mathrm{LM}$ & LM & 3 & (1)(2)(3)(6) \\
\hline Chen YM 2017 [25] & $50(31 / 19)$ & $50(30 / 20)$ & BBR 0.5 tid & Met 0.25 tid & 6 & (1)(2)(6) \\
\hline
\end{tabular}

Note: -, no record; BBR, berberine; LM, lifestyle modification; Met, metformin; (1) FPG; (2) 2hPG; (3) HbAlc; (4) HOMA-IR; (5) HOMA- $\beta$; (6) BMI; (7) adverse effects. 
random sequence generation methods, such as random number tables [20] [23]. There was insufficient information to determine whether the randomizations were carried out correctly in the rest of the studies. Only one study described the allocation concealment [22], one study used a single blind [22], and three studies reported the number of withdrawals and drop-outs in each group [19] [22] [23]. None of the studies indicated any other bias. The risk of bias in included studies is shown in Figure 2.

\subsection{Outcome Indicators}

Seven studies (nine trials) were included in the study. The control groups were all lifestyle modification or metformin treatment. Considering that the intervention measures of berberine treatment are different, the influencing factors such as drug dosage and course of treatment can not be combined and analyzed. Therefore, the intervention types of experimental and control groups were analyzed in subgroups, which were divided into berberine vs. lifestyle and berberine vs. metformin. Because some data in Wang L [23] article, our previous research results, were abnormal distribution, the meta analysis was carried out on the basis of the original data.

\subsubsection{Efficacy of Berberine Treatment on FPG}

There were six trials that compared the effect of berberine vs. lifestyle on FPG and two trials for berberine vs. metformin. Due to high heterogeneity, $P>50 \%$, random-effect (RE) model was used for the analysis. Subgroup analysis showed that berberine significantly reduced FPG level compared with lifestyle group, $[\mathrm{MD}=-0.39,95 \% \mathrm{CI}(-0.63,-0.16), P=0.001]$. There was no significant difference between berberine and metformin, $[\mathrm{MD}=-0.01,95 \% \mathrm{CI}(-0.08,0.05)$, $P=0.71]$. See Figure 3 .

\subsubsection{Efficacy of Berberine Treatment on $2 \mathrm{hPG}$}

There were six trials that compared the effect of berberine vs. lifestyle on 2hPG and two trials for berberine vs. metformin. Due to high heterogeneity, $P>50 \%$, random-effect (RE) model was used for the analysis. Subgroup analysis showed

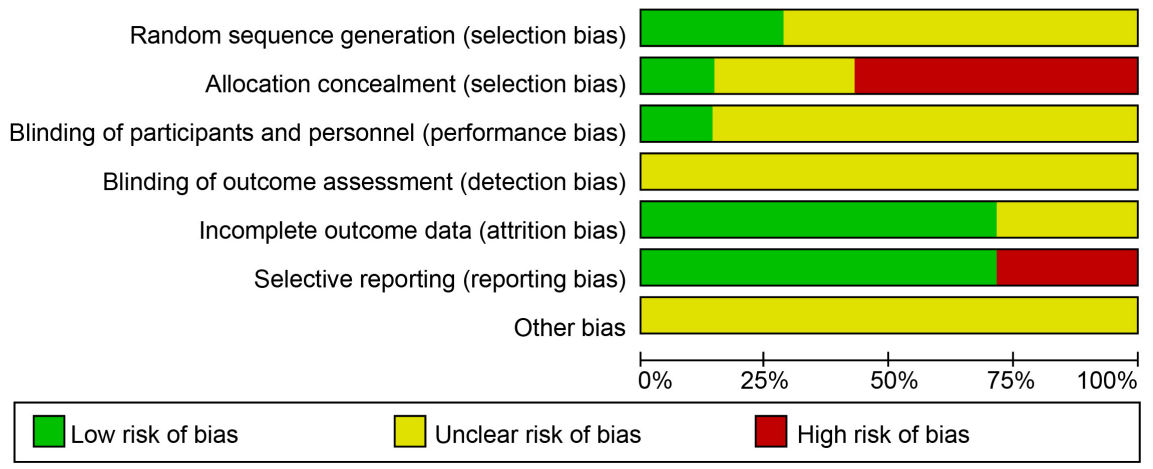

Figure 2. Risk of bias accessed using RevMan 5.4 according to the guidance in the Cochrane Handbook. Green represents low risk of bias, yellow represents unclear risk of bias, and red represents high risk of bias. 


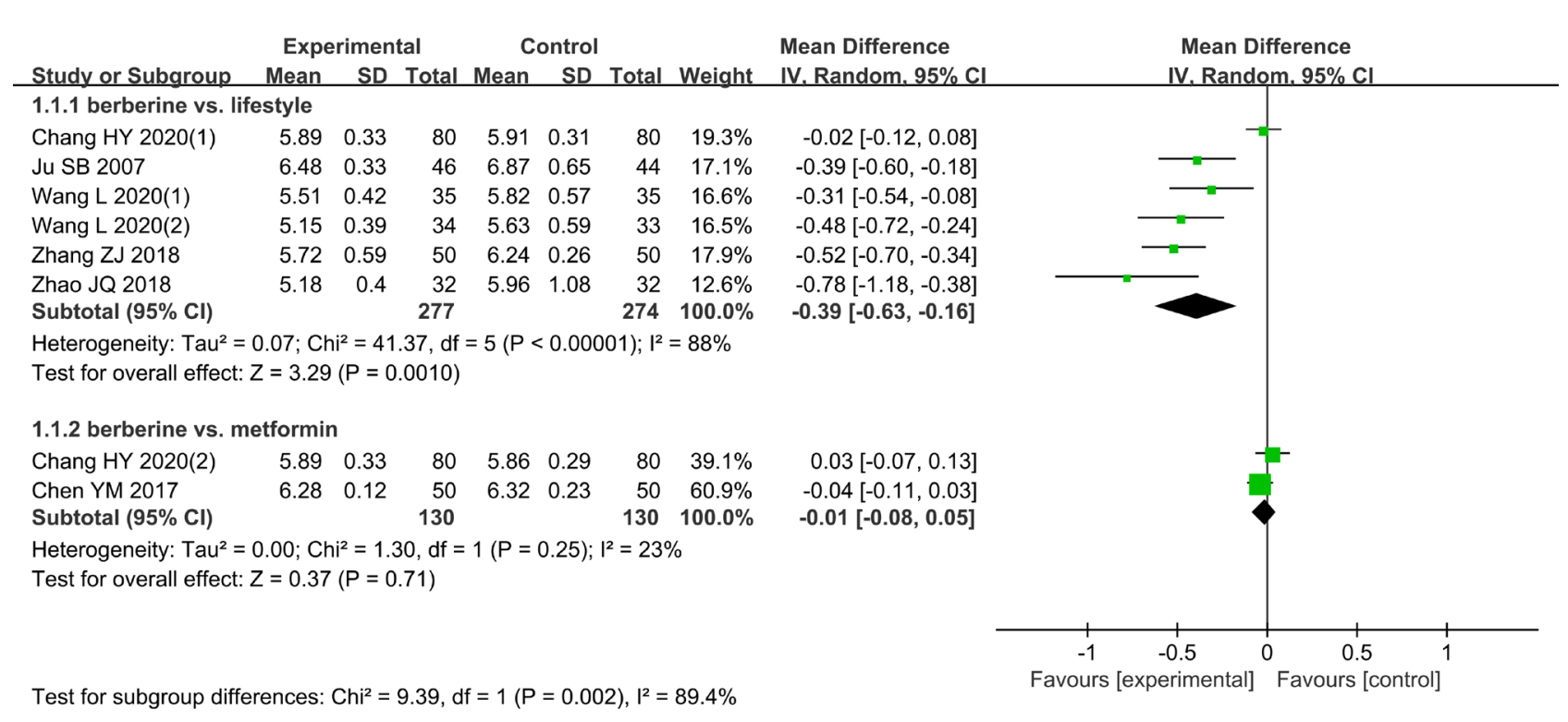

Figure 3. Forest plot of outcome measure FPG.

that berberine significantly reduced $2 \mathrm{hPG}$ leval compared with lifestyle group, $[\mathrm{MD}=-1.51,95 \% \mathrm{CI}(-2.43,-0.59), P=0.001]$. There was no significant difference between berberine and metformin, [MD $=-0.07,95 \% \mathrm{CI}(-0.29,0.14)$, $P=0.51]$. See Figure 4 .

\subsubsection{Efficacy of Berberine Treatment on HbA1c}

There were six trials that compared the effect of berberine vs. lifestyle on HbAlc and two trials for berberine vs. metformin. Due to high heterogeneity, $\vec{P}>50 \%$, random-effect (RE) model was used for the analysis. Subgroup analysis showed that berberine significantly reduced HbAlc level compared with lifestyle group, $[\mathrm{MD}=-0.20,95 \% \mathrm{CI}(-0.32,-0.08), P=0.002]$. There was no significant difference between berberine and metformin, [MD $=-0.03,95 \% \mathrm{CI}(-0.08,0.02), P=$ 0.22]. See Figure 5.

\subsubsection{Efficacy of Berberine Treatment on HOMA-IR}

There were four trials that compared the effect of berberine vs. Lifestyle on HOMA-IR and one trial for berberine vs. metformin. Due to high heterogeneity, $I^{2}>50 \%$, random-effect (RE) model was used for the analysis. There was no significant difference between berberine and lifestyle or metformin, $[\mathrm{MD}=-0.13$, $95 \% \mathrm{CI}(-0.33,0.06), P=0.18]$ and $[\mathrm{MD}=-0.01,95 \% \mathrm{CI}(-0.05,0.03), P=$ $0.64]$, respectively. See Figure 6.

\subsubsection{Efficacy of Berberine Treatment on HOMA- $\beta$}

One trial compared the effect of berberine vs. lifestyle on HOMA- $\beta$. There was no significant difference between the two groups, $[\mathrm{MD}=0.17,95 \% \mathrm{CI}(-0.03$, 0.37), $P=0.09]$. See Figure 7 .

\subsubsection{Efficacy of Berberine Treatment on BMI}

There were four trials that compared the effect of berberine vs. lifestyle on BMI 


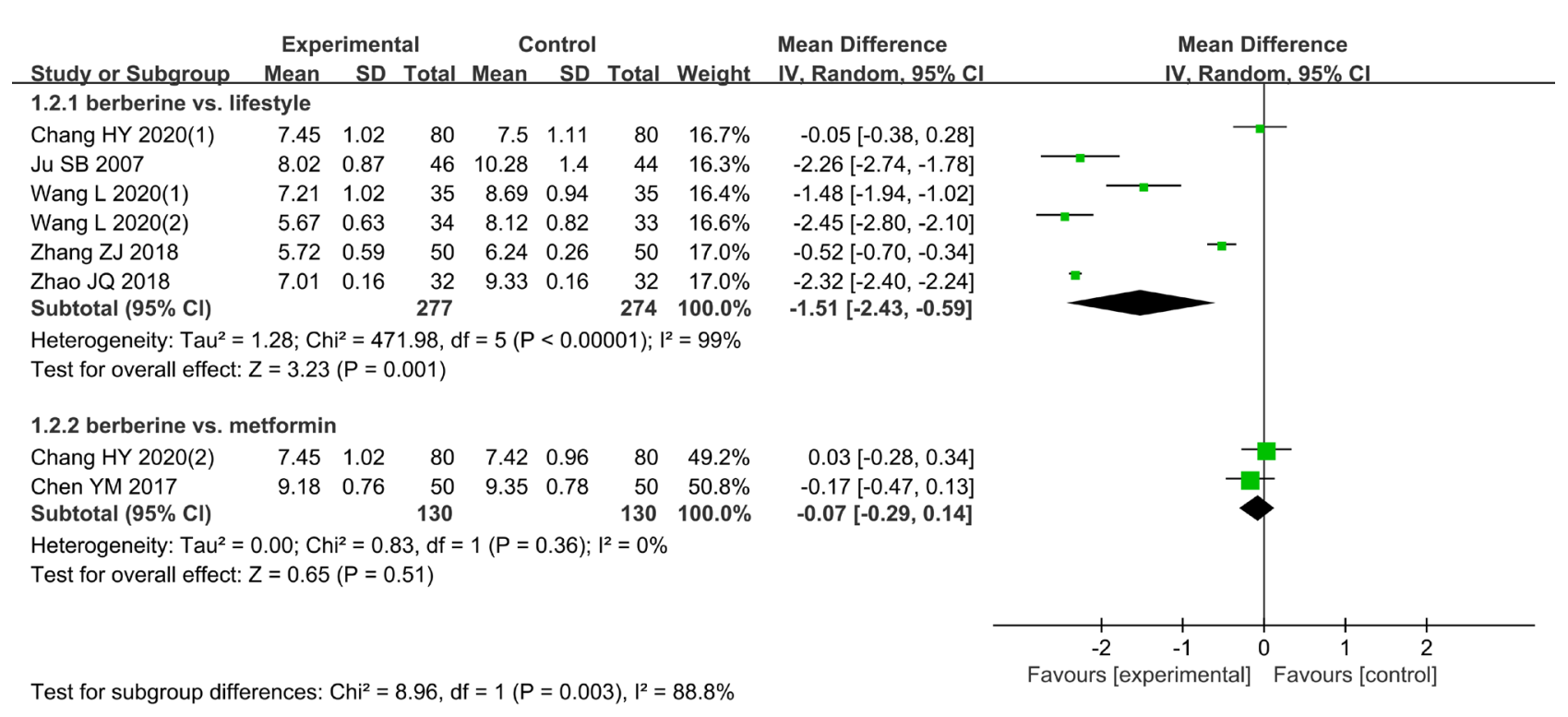

Figure 4. Forest plot of outcome measure 2hPG.

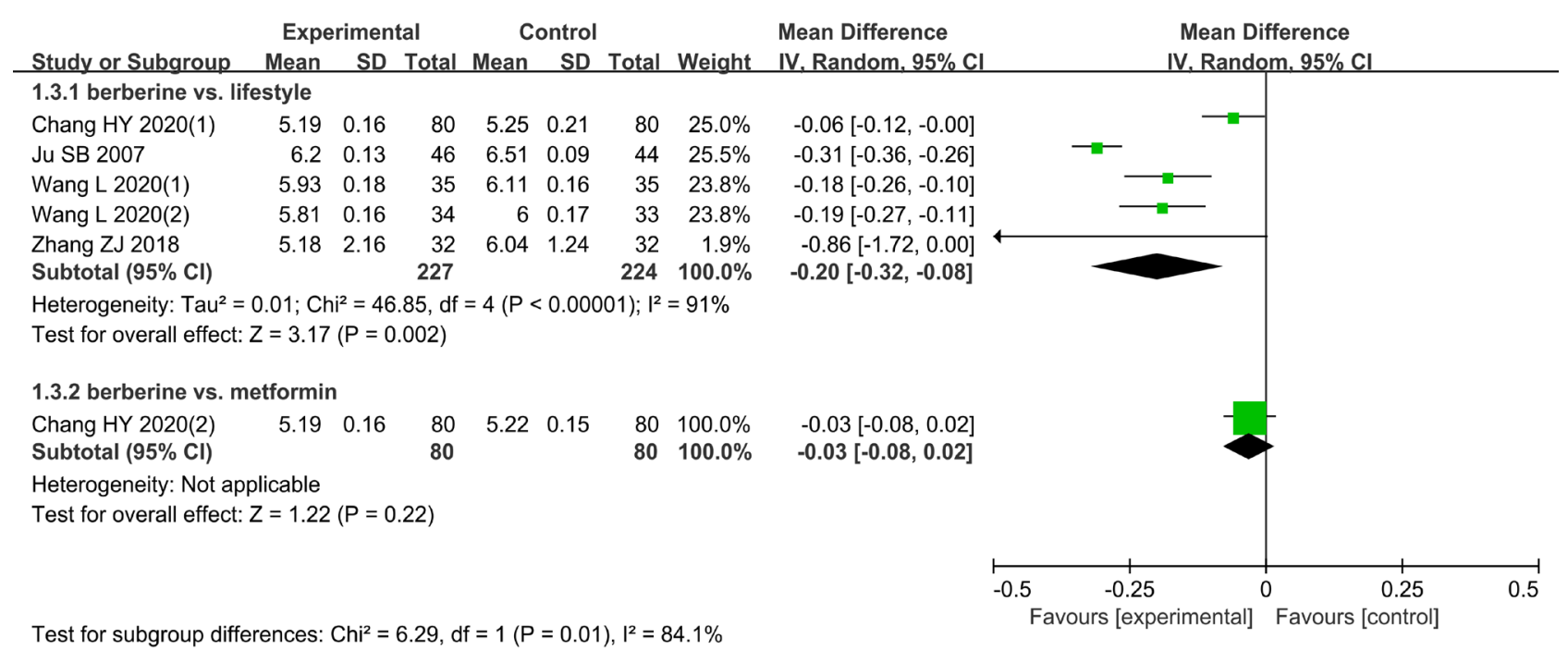

Figure 5. Forest plot of outcome measure HbAlc.

and one trial for berberine vs. metformin. Due to high heterogenerty, $\vec{P}>50 \%$, random-effect (RE) model was used for the analysis. There was no significant difference between berberine and lifestyle or metformin, $[\mathrm{MD}=-1.14,95 \% \mathrm{CI}$ $(-2.52,0.25), P=0.11]$ and $[\mathrm{MD}=-0.45,95 \% \mathrm{CI}(-1.63,0.73), P=0.45]$, respectively. See Figure 8.

\subsubsection{Efficacy of Berberine Treatment on Adverse Effects}

Five trials reported the number of adverse effects and the other trials only stated slight adverse effects of berberine without clear data. Due to low heterogeneity, $I^{2}<50 \%$, fixed-effect (FE) model was used for the analysis. Subgroup analysis showed that there was no significant difference between berberine and lifestlye, $[\mathrm{MD}=3.75,95 \% \mathrm{CI}(0.61,23.2), P=0.15]$. Compared with metformin, the 


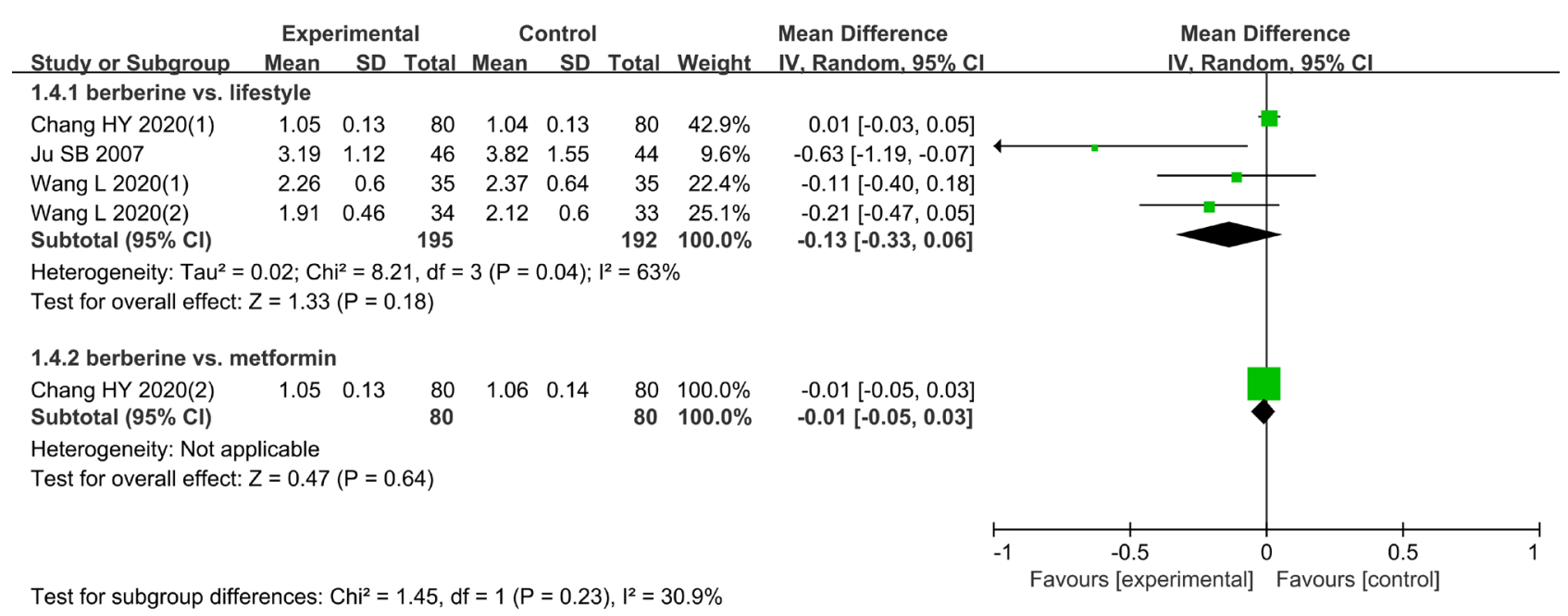

Figure 6. Forest plot of outcome measure HOMA-IR.

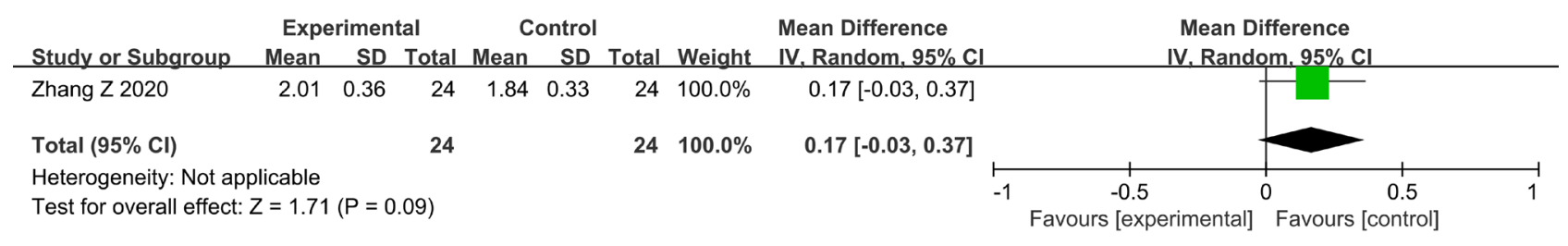

Figure 7. Forest plot of outcome measure HOMA- $\beta$.

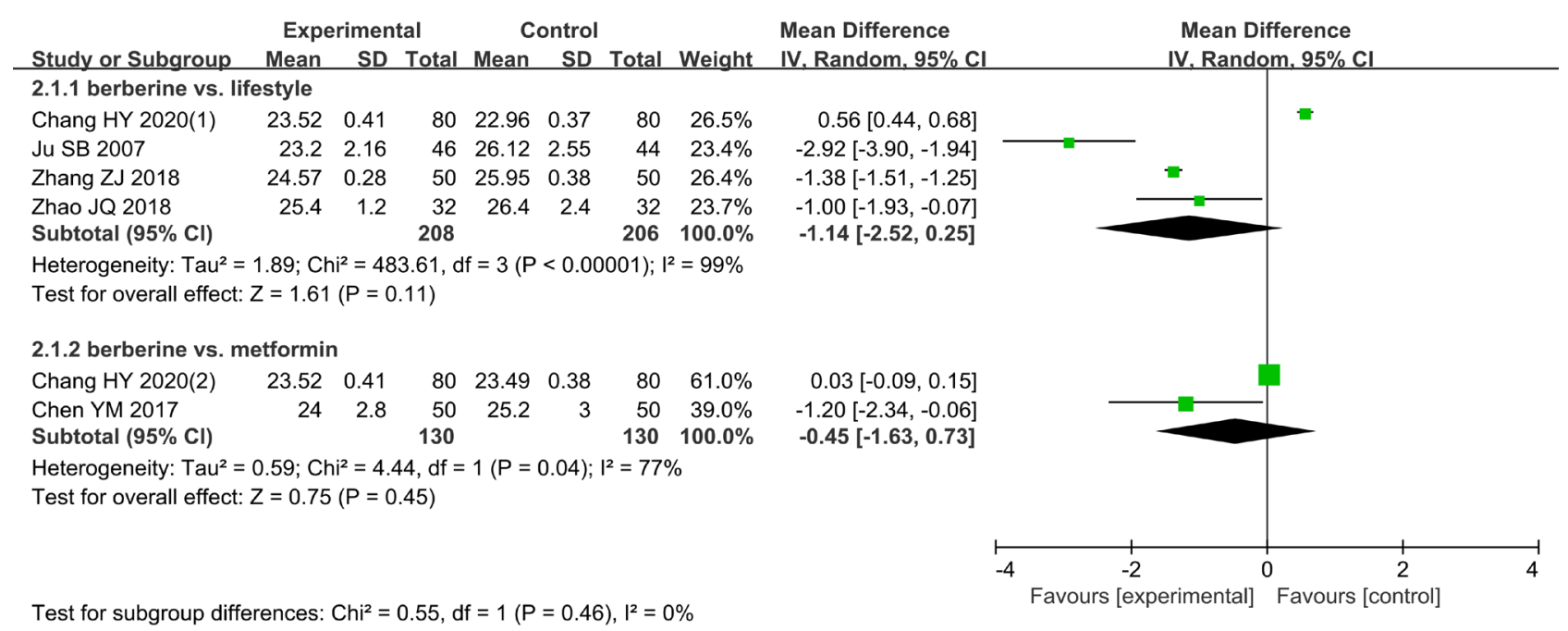

Figure 8. Forest plot of outcome measure BMI.

adverse effects rate of berberine was significantly decreased, [MD $=0.11,95 \% \mathrm{CI}$ $(0.01,0.93), P=0.04]$. All reported events were mild, including constipation, diarrhea, nausea and abdominal distension. No serious adverse effects from berberine were reported. See Figure 9.

\subsection{Sensitivity Analysis}

Sensitivity analysis was carried out by eliminating literature one by one. FPG, 


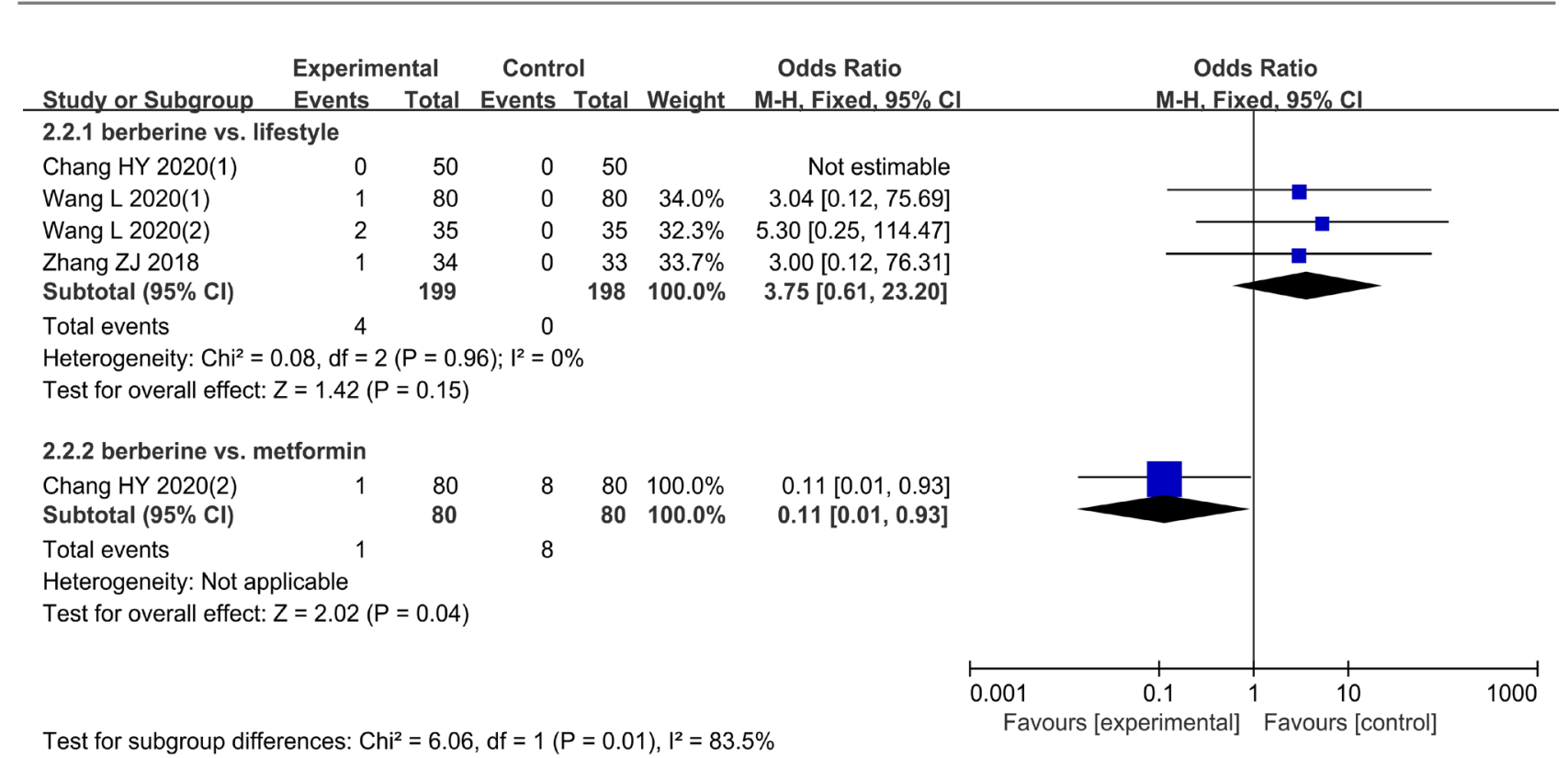

Figure 9. Forest plot of adverse effects.

HOMA-IR and BMI for the subgroup of berberine vs. Lifestyle, were significantly affected by the article, Chang HY [21]. The results changed from $[P=$ $0.001, P=88 \%, \mathrm{MD}=-0.39,95 \% \mathrm{CI}(-0.63,-0.16)],[P=0.18, P=63 \%, \mathrm{MD}=$ $-0.13,95 \% \mathrm{CI}(-0.33,0.06)]$ and $\left[P=0.11, \mathrm{I}^{2}=99 \%, \mathrm{MD}=-1.14,95 \% \mathrm{CI}\right.$ $(-2.52,0.25)]$ to $\left[P=0.00001, P^{2}=19 \%, \mathrm{MD}=-0.46,95 \% \mathrm{CI}(-0.58,-0.35)\right]$, $\left[P=0.04, P^{2}=23 \%, \mathrm{MD}=-0.23,95 \% \mathrm{CI}(-0.44,-0.01)\right]$ and $\left[P=0.0002, P^{2}=\right.$ $80 \%, \mathrm{MD}=-1.71,95 \% \mathrm{CI}(-2.60,-0.81)]$, respectively. After excluding the article, berberine could also significantly reduce HOMA-IR and BMI of prediabetes.

\subsection{Publication Bias Analysis}

Owing to the limited number (below ten) of trials included in each analysis, publication bias was not assessed.

\section{Discussion}

\subsection{Summary and Analysis of Evidence}

There have been a lot of clinical studies or reports about berberine in the treatment of T2DM. But it is relatively few studies for prediabetes. At present, no meta-analysis of the efficacy and safety of berberine in prediabetes has been done. In this systematic review, we selected seven clinical studies and the strategy follows the Preferred Reporting Items for Systematic Reviews and Meta-Analyses (PRISMA) statement. A total of 859 participants were involved, of which 431 and 428 were in the experimental and control groups. Our findings upon the seven studies showed that compared with lifestyle group, berberine could lower the level of FPG [MD $=-0.39,95 \%$ CI $(-0.63,-0.16), P=0.001]$, $2 \mathrm{hPG}[\mathrm{MD}=-1.51,95 \% \mathrm{CI}(-2.43,-0.59), P=0.001]$ and HbA1c $[\mathrm{MD}=-0.20$, 
95\% CI $(-0.32,-0.08), P=0.002]$. There was no statistical significance between berberine and metformin. In addition, berberine evaluated in our review generally appeared to be safe. The adverse effects were commonly gastrointestinal discomforts including constipation, diarrhea, nausea and abdominal distension. No serious adverse effects from berberine were reported.

In order to minimize the heterogeneity, we used the subgroup analysis according to different interventions. However, when FPG, 2hPG, HbA1c, HOMI-IR and BMI data were aggregated in the subgroup of berberine vs. lifestyle, the heterogeneity was still high. Then we used the method of eliminating references one by one to carry out the sensitivity analysis. It was found that Chang HY, 2020(1) [21] had the greatest impact on the results. Berberine could also significantly reduce HOMA-IR $\left[P=0.04, P^{2}=23 \%, \mathrm{MD}=-0.23,95 \% \mathrm{CI}(-0.44\right.$, $-0.01)]$ and BMI $\left[P=0.0002, P^{2}=80 \%, \mathrm{MD}=-1.71,95 \% \mathrm{CI}(-2.60,-0.81)\right]$ after excluding the article. The reason may be that the doses of berberine in this study were significantly lower than those in other studies.

\section{2. limitations}

This analysis also has several limitations. All the included studies were conducted among Chinese participants in the mainland of China. There was a high risk of selection bias. Although all the studies mentioned random allocation, five studies did not describe the generation of random sequences, and most of the studies did not describe adequate allocation concealment. Only one study described single blindness. Three studies reported withdrawals and drop-outs. So it may lead to selection bias and implementation bias. Potential bias in selection of patients (such as age, gender or blood glucose level at baseline), administration of treatment and assessment of outcomes could lead to overestimation of the therapeutic efficacy of berberine. Moreover, the research approaches of the trials were not described or published in advance. These may lead to follow-up bias and reporting bias. Owing to the limited number (below ten) of trials included in each analysis, publication bias was not assessed. Therefore all of the outcomes should be carefully interpreted based on substantial methodological and clinical diversity.

\subsection{Inspiration}

This study suggests that the methodological quality of berberine in the treatment of prediabetes is generally low, which may lower the internal authenticity of the results, then affect their external authenticity. High quality RCTs should be carried out, especially scientific and reasonable methodological research design. Attentions should be paid to the design and implementation of clinical studies: 1) Register programmes prior to implementation; 2) Estimate sample size before the study; 3) Report detailedly on random sequence generation, allocation concealment and blinding of participants, researchers and evaluators; 4) Report results and analyze reasons of withdrawals and drop-outs; 5) Large sample sizes 
and long-term follow-up are needed to the evaluation of the efficacy and safety of berberine; 6) Report strictly according to CONSORT [26] to improve the levels of evidence and clinical values.

\section{Conclusion}

This study indicates that Berberine has good efficacy and safety in the treatment of prediabetes. Due to the quality limitations of the included trials, the above conclusions need to be further verified by high-quality, large sample size and multi-center RCTs.

\section{Conflicts of Interest}

None of the authors has any potential conflicts of interest associated with this research.

\section{Funding}

This research was supported by a grant from the project of Administration of the Traditional Chinese Medicine of Guangdong Province of China (Grant No.20191065).

\section{References}

[1] Chinese Society of Endocrinology, Chinese Diabetes Society, Chinese Endocrinologist Association, et al. (2020) Intervention for Adults with Pre-Diabetes: A Chinese Expert Consensus. Chinese Journal of Endocrinology and Metabolism, 36, 371-380. http://doi.org/10.3760/cma.j.cn311282-20200115-00022

[2] Li, Y., Teng, D., Shi, X., et al. (2020) Prevalence of Diabetes Recorded in Mainland China Using 2018 Diagnostic Criteria from the American Diabetes Association: National Cross Sectional Study. BMJ, 369, 1-11. https://doi.org/10.1136/bmj.m997

[3] Glauber, H., Vollmer, W. and Nichols, G. (2018) A Simple Model for Predicting Two-Year Risk of Diabetes Development in Individuals with Prediabetes. The Permanente Journal, 22, 17-50. http://doi.org/10.7812/TPP/17-050

[4] Bigna, J., Nansseu, J., Katte, C., et al. (2018) Prevalence of Prediabetes and Diabetes Mellitus among Adults Residing in Cameroon: A Systematic Review and $\mathrm{Me}-$ ta-Analysis. Diabetes Research and Clinical Practice, 137, 109-118. https://doi.org/10.1016/j.diabres.2017.12.005

[5] Chinese Society of Endocrinology (2014) Intervention for Adults with Type 2 Diabetes: A Chinese Expert Consensus. Chinese Journal of Endocrinology and Metabolism, 30, 277-283.

[6] Malin, S.K., Liu, Z., Barrett, E.J., et al. (2016) Exercise Resistance across the Prediabetes Phenotypes: Impact on Insulin Sensitivity and Substrate Metabolism. Reviews in Endocrine and Metabolic Disorders, 17, 81-90. https://doi.org/10.1007/s11154-016-9352-5

[7] Pan, X.R., Li, G.W., Hu, Y.H., et al. (1995) Effect of Dietary and/or Exercise Intervention on Incendence of Diabetes in 530 Subjects with Impared Glucose Tolerance from 1986-1992. Chinese Journal of Internal Medicine, 34, 108-112.

[8] China Pre-Diabetes Clinical Intervention Expert Group (2019) Chinese Experts Consensus of Clinical Intervention for Pre-Diabetes (Draft for Comments). Drug 
Evaluation, 16, 3-16.

[9] Fang, C.H., Zhao, J.D., Shi, G.B., et al. (2014) Comprehensive Scheme of Prevention and Treatment with TCM in PiDan (Prophase of Diabetes) and Clinical Research. Tianjin Journal of Traditional Chinese Medicine, 31, 583-587.

[10] You, L.Z., Yu, D.D., Fang, C.H., et al. (2020) Clinical Research of Pre-Diabetes Based on Evidence-Based Chinese Medicine. China Journal of Traditional Chinese Medicine and Pharmacy, 35, 1343-1346.

[11] Zhang, L.Z. and Ji, Y.F. (1999) The Application of Berberine in the Treatment of Diabetes. Journal of Practical Traditional Chinese Internal Medicine, 13, 22. http://doi.org/10.13729/j.issn.1671-7813.1999.03.019

[12] Kong, W., Wei, J., Abidi, P., et al. (2004) Berberine is a Novel Cholesterol-Lowering Drug Working through a Unique Mechanism Distinct from Statins. Nature Medicine, 10, 1344-1351. https://doi.org/10.1038/nm1135

[13] Lee, Y.S., Kim, K.H., Yoon, M.J., et al. (2006) Berberine, a Natural Plant Product, Activates AMP-Activated Protein Kinase With Beneficial Metabolic Effects in Diabetic and Insulin-Resistant States. Diabetes, 55, 2256-2264.

https://doi.org/10.2337/db06-0006

[14] KÜpeli, E., Kosar, M., Yesilada, E., et al. (2002) A Comparative Study on the Anti-Infl Ammatory, Antinociceptive and Antipyretic Effects of Isoquinoline Alkaloids from the Roots of Turkish Berberis Species. Life Science, 72, 645-657.

[15] World Health Organization (1999) Definition, Diagnosis and Classification of Diabetes Mellitus and Its Complications: Report of a WHO Consultation. Part 1, Diagnosis and Classification of Diabetes Mellitus. World Health Organization, Geneva.

[16] Chinese Diabetes Society. (2014) Guidelines for the Prevention and Treatment of Type 2 Diabetes in China (2013). Chinese Journal of Endocrinology and Metabolism, 30, 893-942. http://doi.org/10.3760/cma.j.issn.1000-6699.2014.10.020

[17] American Diabetes Association. (2011) Diagnosis and Classification of Diabetes Mellitus. Diabetes Care, 34, S62-S69. https://doi.org/10.2337/dc11-S062

[18] Higgins, J.P. and Green, S. (2019) Cochrane Handbook for Systematic Review of Interventions. Versin 5.1.0 [EB/OL]. http://www.cochrane-handbook.org

[19] Ju, S.B., Tan, L.R., Su, W., et al. (2007) Interventioal Effect of Berberine Liposome on Impaired Glucose Tolerance Accompanied with Hyperlipemia. Journal of Practical Traditional Chinese Medicine, 23, 490-492.

[20] Zhang, Z.J., Lu, Q.Y., Hang, T, et al. (2018) Clinical Observation of Berberine in Patients with Impaired Fasting Glucose. Zhejiang Journal of Traditional Chinese Medicine, 53, 393-394. http://doi.org/10.13633/j.cnki.zjtcm.2018.06.002

[21] Chang, H.Y., Xu, L.P., Cheng, T.J., et al. (2020) Effect of Lifestyle Intervetion and Drug Treatment on Pre-Diabetes Lesions. Front Endocrinol, 35, 920-925.

[22] Zhang, Z. and Zhou, W.D. (2020) The Effect of Berberine on First Phase Insulin Secretion in Patients with Impaired Glucose Tolerance. Journal of practical diabetology, 16, 27-28.

[23] Wang, L., Ge, H., Wei, G.H., et al. (2020) Berberine and Prediabetes: A Clinical Observational Study. Journal of Diabetes Mellitus, 10, 209-221. https://doi.org/10.4236/jdm.2020.104017

[24] Zhao, J.Q., Lv, Q.B. and Zhang, C.Z. (2018) The Clinical Study of Berberine Intervention to Delay the Development of Diabetes Mellitus in Prediabetic Population. Journal of Practical Diabetology, 14, 31-33. 
[25] Chen, Y.M., Sun, G.P. and Sun, Y.G. (2017) Effect of Berberine on Weight and Outcome after Treatment in Obese Prediabetic Patients. Chinese Journal of Prevention and Control of Chronic Disease, 25, 54-56.

http://doi.org/10.16386/j.cjpccd.issn.1004-6194.2017.01.015

[26] Bian, Z., Liu, B., Moher, D., et al. (2011) Consolidated Standards of Reporting Trials (CONSORT) for Traditional Chinese Medicine: Current Situation and Future Development. Frontiers of Medicine, 5, 171-177.

https://doi.org/10.1007/s11684-011-0132-Z 